\title{
Standardized Glycemic Management with a Computerized Workflow and Decision Support System for Hospitalized Patients with Type 2 Diabetes on Different Wards
}

\author{
Katharina M. Neubauer, BSc, MSc, Julia K. Mader, MD, Bernhard Höll, BSc, MSc, \\ Felix Aberer, MD, Klaus Donsa, BSc, MSc, ${ }^{2}$ Thomas Augustin, PhD, \\ Lukas Schaupp, PhD, Stephan Spat, MSc, ${ }^{2}$ Peter Beck, PhD, \\ Friedrich M. Fruhwald, MD, ${ }^{3}$ Christian Schnedl, MD, Alexander R. Rosenkranz, MD, \\ David B. Lumenta, MD, ${ }^{5}$ Lars-Peter Kamolz, MD, MSc, Johannes Plank, MD, \\ and Thomas R. Pieber, MD ${ }^{1,2}$
}

\begin{abstract}
Background: This study investigated the efficacy, safety, and usability of standardized glycemic management by a computerized decision support system for non-critically ill hospitalized patients with type 2 diabetes on four different wards.

Materials and Methods: In this open, noncontrolled intervention study, glycemic management of 99 patients with type 2 diabetes (62\% acute admissions; 41 females; age, $67 \pm 11$ years; hemoglobin A1c, $65 \pm 21 \mathrm{mmol} /$ mol; body mass index, $30.4 \pm 6.5 \mathrm{~kg} / \mathrm{m}^{2}$ ) on clinical wards (Cardiology, Endocrinology, Nephrology, Plastic Surgery) of a tertiary-care hospital was guided by GlucoTab ${ }^{\circledR}$ (Joanneum Research GmbH [Graz, Austria] and Medical University of Graz [Graz, Austria]), a mobile decision support system providing automated workflow support and suggestions for insulin dosing to nurses and physicians.

Results: Adherence to insulin dosing suggestions was high (96.5\% bolus, $96.7 \%$ basal). The primary outcome measure, percentage of blood glucose (BG) measurements in the range of $70-140 \mathrm{mg} / \mathrm{dL}$, occurred in $50.2 \pm 22.2 \%$ of all measurements. The overall mean BG level was $154 \pm 35 \mathrm{mg} / \mathrm{dL}$. BG measurements in the ranges of $60-70 \mathrm{mg} / \mathrm{dL}, 40-60 \mathrm{mg} / \mathrm{dL}$, and $<40 \mathrm{mg} / \mathrm{dL}$ occurred in $1.4 \%, 0.5 \%$, and $0.0 \%$ of all measurements, respectively. A regression analysis showed that acute admission to the Cardiology Ward $(+30 \mathrm{mg} / \mathrm{dL})$ and preexisting home insulin therapy $(+26 \mathrm{mg} / \mathrm{dL})$ had the strongest impact on mean BG. Acute admission to other wards had minor effects $(+4 \mathrm{mg} / \mathrm{dL})$. Ninety-one percent of the healthcare professionals felt confident with GlucoTab, and $89 \%$ believed in its practicality and $80 \%$ in its ability to prevent medication errors.

Conclusions: An efficacious, safe, and user-accepted implementation of GlucoTab was demonstrated. However, for optimized personalized patient care, further algorithm modifications are required.
\end{abstract}

Divisions of ${ }^{1}$ Endocrinology and Metabolism, ${ }^{3}$ Cardiology, and ${ }^{4}$ Nephrology, Department of Internal Medicine, Medical University of Graz, Graz, Austria.

${ }^{2}$ Joanneum Research GmbH, HEALTH, Institute for Biomedicine and Health Sciences, Graz, Austria.

${ }^{5}$ Division of Plastic, Aesthetic and Reconstructive Surgery, Department of Surgery, Medical University of Graz, Graz, Austria.

This study is registered at Clinicaltrials.gov with clinical trial registration number NTC01932775.

Parts of this study were presented at the $74^{\text {th }}$ Scientific Sessions of the American Diabetes Association, held in San Francisco, California, June 3-17, 2014, and the 14 ${ }^{\text {th }}$ Annual Diabetes Technology Meeting, held in Bethesda, Maryland, November 6-8, 2014. Parts of the continuous glucose monitoring (CGM) data were previously published. ${ }^{1}$

(C) The Author(s) 2015; Published by Mary Ann Liebert, Inc. This Open Access article is distributed under the terms of the Creative Commons Attribution Noncommercial License (http://creativecommons.org/licenses/by-nc/4.0/) which permits any noncommercial use, distribution, and reproduction in any medium, provided the original author(s) and the source are credited. 


\section{Background}

$\mathbf{U}$ P TO 35\% OF ALL HOSPITALIZED PATIENTS suffer from diabetes, ${ }^{2,3}$ and hospital management costs for these patients place a serious financial burden to public healthcare systems. ${ }^{4}$ In addition, patients with diabetes have an increased risk of infections, ${ }^{5}$ prolonged hospital stays, and increased mortality due to insufficient insulin dosing management, which is caused by a varying degree of knowledge on glycemic control, clinical inertia, and the fear of hypoglycemia. ${ }^{6}$ Considerable efforts have been made to improve glycemic management regarding blood glucose (BG) measurements, but an adequate insulin therapy in clinical practice is still lacking in many hospitals. ${ }^{6,7}$

Guidelines have been developed to improve glycemic management in hospitals that recommend a target range of less than $140 \mathrm{mg} / \mathrm{dL}$ for premeal BG and less than $180 \mathrm{mg} / \mathrm{dL}$ for a random $\mathrm{BG}$ measurement for non-critically ill patients treated with insulin. ${ }^{8,9}$ These target ranges should be achievable by scheduled subcutaneous insulin dosing with basal, nutritional, and a correctional component. ${ }^{8,9}$ The guidelines also suggest the development and evaluation of evidence-based computerized decision support systems, including computerized insulin and BG data display that will not only improve glycemic control but also workflow and communication among healthcare professionals. ${ }^{6}$

Paper-based algorithms for basal bolus insulin therapy have been developed that increase the quality of glycemic control and reduce hospital complications. ${ }^{10-12}$ Within the framework of a European Commission-funded project (FP7 248590), we have modified and tested standardized recommendations of a paper-based insulin dosing algorithm to comply with daily workflow requirements on general wards. ${ }^{13}$ This modified algorithm was then implemented in a mobile decision support system for basal bolus insulin dosing, the GlucoTab ${ }^{\circledR}$ system (Joanneum Research GmbH [Graz, Austria] and Medical University of Graz [Graz, Austria]), which was subsequently customized and tested in a clinical study with 30 patients. ${ }^{14}$

In the current study, the final mobile version of the GlucoTab system was used for the first time to guide the glycemic management process on four different general wards in the Departments of Internal Medicine and Surgery. The purpose of this study was to investigate the efficacy, safety, and usability of a standardized glycemic management with the GlucoTab system for non-critically ill patients with type 2 diabetes mellitus.

\section{Materials and Methods}

This study was an open, noncontrolled interventional study in hospitalized patients with type 2 diabetes mellitus. The study was conducted on four general wards of a tertiarycare hospital (Medical University of Graz). The participating wards were Endocrinology, Cardiology, Nephrology and Plastic Surgery, which are each independently managed by the respective division. All patients gave written informed consent prior to any study activity, and the study was approved by the ethical board of Medical University of Graz (protocol number EK-No. 25-344 ex 12/13). This study was conducted in full accordance with the principles of the Declaration of Helsinki.

\section{Patient characteristics}

The GlucoTab system was subsequently implemented on the four participating general wards. In total, 99 hospitalized patients were competitively recruited from May 2013 to December 2013. Hospitalized patients who met the inclusion criteria were included in the study after they consented to participate. The demographic and clinical characteristics of the study participants are presented in Table 1. Inclusion criteria were as follows: age $\geq 18$ years and type 2 diabetes (treated with diet, oral antidiabetes drugs, non-insulininjected antidiabetes drugs, insulin therapy, or any combination of the four therapies) or newly diagnosed hyperglycemia requiring subcutaneous insulin therapy. Patients were switched to insulin therapy in the case of hyperglycemia judged by the treating physician according to evidence-based recommendations to use insulin therapy as the preferred method for glycemic control in hospitalized patients. ${ }^{8,9}$ Glycemic management with the GlucoTab system was not performed for patients with the following exclusion criteria: type $1 \mathrm{di}-$ abetes, gestational diabetes, any condition which the investigator or treating physician felt would interfere with the study or the safety of the patient, pregnancy, any mental condition rendering the patient incapable of giving consent, known or suspected allergy to insulin glargine or insulin aspart, continuous parenteral nutrition, or participation in another study that could interfere with this study.

\section{Standardized glycemic management with GlucoTab}

GlucoTab is a mobile computerized clinical decision support system for subcutaneous insulin therapy that supports nurses and physicians in glycemic management of hospitalized patients in two main tasks: First, it assists clinical healthcare professionals in organizing the treatment workflow of patients with type 2 diabetes mellitus by providing automated workflow support, including display for open tasks, facilitating documentation and providing visualization of BG values, nutrition and insulin doses. Second, it provides two standardized recommendations based on a basal-bolus insulin titration protocol ${ }^{10-12,14}$ for (1) the total daily insulin dose, which is prescribed by the treating physician during the ward round, and (2) insulin dose suggestions for individual insulin administrations before each meal, at bedtime, and after intermediate BG measurements, if required. After confirmation of the suggested insulin dosage, the insulin is injected subcutaneously by an authorized nurse.

The standardized recommendations for insulin dose calculation, based on the modified basal bolus insulin titration protocol, ${ }^{10-12,14}$ consist of a daily dose of basal insulin (insulin glargine; Sanofi-Aventis, Frankfurt am Main, Germany), bolus insulin (insulin aspart; NovoNordisk, Bagsværd, Denmark) before each meal, and a correctional dose at bedtime to achieve fasting and premeal BG values of less than $140 \mathrm{mg} /$ dL. ${ }^{8,9}$ Insulin therapy was started with a total daily dose of 0.5 units $/ \mathrm{kg}$ of body weight. The initial total daily dose was reduced to 0.3 units $/ \mathrm{kg}$ of body weight in patients $\geq 70$ years of age and/or with creatinine values of $\geq 2.0 \mathrm{mg} / \mathrm{dL}$. In case the patient had already been on insulin therapy, the protocol allowed use of the former total insulin dose as the initial dose, which could be adjusted by the treating physician. One-half of the total daily dose was administered as basal insulin once a day before lunch. The other half was administered as bolus 
Table 1. Clinical Characteristics of the Study Population

\begin{tabular}{|c|c|c|c|c|c|c|}
\hline Variable & $\begin{array}{c}\text { Total } \\
(\mathrm{n}=99)\end{array}$ & $\begin{array}{l}\text { Nephrology } \\
\quad(\mathrm{n}=15)\end{array}$ & $\begin{array}{l}\text { Cardiology } \\
\quad(\mathrm{n}=30)\end{array}$ & $\begin{array}{l}\text { Endocrinology } \\
\qquad(\mathrm{n}=42)\end{array}$ & $\begin{array}{l}\text { Plastic surgery } \\
\quad(\mathrm{n}=12)\end{array}$ & $\mathrm{P}$ \\
\hline Gender, female $[n(\%)]$ & $41 / 41$ & $3 / 20$ & $12 / 40$ & $20 / 48$ & $6 / 50$ & 0.28 \\
\hline Ethnicity (Caucasian/African) & $98 / 1$ & $15 / 0$ & $30 / 0$ & $42 / 0$ & $11 / 1$ & 0.13 \\
\hline Age (years) & $67 \pm 11$ & $64 \pm 8$ & $70 \pm 12$ & $67 \pm 11$ & $65 \pm 10$ & 0.31 \\
\hline Body mass index $\left(\mathrm{kg} / \mathrm{m}^{2}\right)$ & $30.4 \pm 6.5$ & $31.0 \pm 5.4$ & $29.4 \pm 6.8$ & $31.1 \pm 6.8$ & $29.4 \pm 6.3$ & 0.57 \\
\hline Weight $(\mathrm{kg})$ & $88 \pm 21$ & $92 \pm 17$ & $84 \pm 24$ & $89 \pm 20$ & $86 \pm 17$ & 0.22 \\
\hline Serum creatinine $(\mathrm{mg} / \mathrm{dL})$ & $1.8 \pm 1.5$ & $3.8 \pm 2.1$ & $1.4 \pm 0.9$ & $1.6 \pm 1.3$ & $1.0 \pm 0.2$ & $<0.05^{\mathrm{a}}$ \\
\hline Renal dialysis $(n)$ & 9 & 6 & 1 & 2 & 0 & - \\
\hline HbA1c & & & & & & 0.13 \\
\hline $\mathrm{mmol} / \mathrm{mol}$ & $65 \pm 21$ & $57 \pm 10$ & $65 \pm 21$ & $70 \pm 24$ & $55 \pm 13$ & \\
\hline$\%$ & $8.1 \pm 4.1$ & $7.4 \pm 3.1$ & $8.1 \pm 4.1$ & $8.6 \pm 4.4$ & $7.2 \pm 3.3$ & \\
\hline Diabetes duration (years) & $13.6 \pm 8.9$ & $13.2 \pm 8.3$ & $11.4 \pm 7.7$ & $15.1 \pm 9.6$ & $13.9 \pm 9.6$ & 0.51 \\
\hline Pre-admission diabetes therapy $[n(\%)]$ & & & & & & 0.60 \\
\hline Diet only & $3(3)$ & $0(0)$ & $1(3)$ & $2(5)$ & $0(0)$ & \\
\hline OAD only & $16(16)$ & $0(0)$ & $6(20)$ & 8 (19) & $2(17)$ & \\
\hline Insulin only & $55(56)$ & $12(80)$ & $13(44)$ & $24(58)$ & $6(50)$ & \\
\hline OAD, GLP-1 analogs & 1 (1) & $0(0)$ & $0(0)$ & $1(2)$ & $0(0)$ & \\
\hline Insulin, OAD & $22(22)$ & $3(20)$ & $9(30)$ & $6(14)$ & $4(33)$ & \\
\hline Insulin, GLP-1 analogs & $2(2)$ & $0(0)$ & $1(3)$ & $1(2)$ & $0(0)$ & \\
\hline Admission type $[n(\%)]$ & & & & & & $<0.05^{\mathrm{b}}$ \\
\hline Planned & $38(38)$ & $6(40)$ & $17(57)$ & 8 (19) & $7(58)$ & \\
\hline Acute & $61(62)$ & $9(60)$ & $13(43)$ & $34(81)$ & $5(42)$ & \\
\hline Admission diagnosis $[n(\%)]$ & & & & & & - \\
\hline Hematological disease & $1(1)$ & $1(7)$ & $0(0)$ & $0(0)$ & $0(0)$ & \\
\hline Gastrointestinal disease & $1(1)$ & $1(7)$ & $0(0)$ & $0(0)$ & $0(0)$ & \\
\hline Endocrine disease & $11(11)$ & $0(0)$ & $1(3)$ & $10(24)$ & $0(0)$ & \\
\hline Cardiovascular disease & $44(44)$ & $4(27)$ & $29(97)$ & $11(26)$ & $0(0)$ & \\
\hline Neurological disease & $1(1)$ & $1(7)$ & $0(0)$ & $0(0)$ & $0(0)$ & \\
\hline Infectious disease & $23(23)$ & $1(7)$ & $0(0)$ & $19(45)$ & $3(25)$ & \\
\hline Renal disease & $8(8)$ & $7(47)$ & $0(0)$ & $1(2)$ & $0(0)$ & \\
\hline Musculoskeletal disease & $9(9)$ & $0(0)$ & $0(0)$ & $1(2)$ & $8(58)$ & \\
\hline Other & $1(1)$ & $0(0)$ & $0(0)$ & $0(0)$ & $1(8)$ & \\
\hline
\end{tabular}

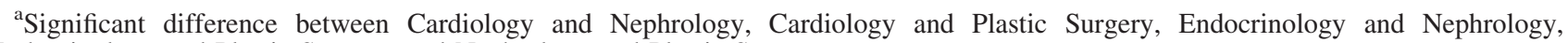
Endocrinology and Plastic Surgery, and Nephrology and Plastic Surgery.

${ }^{\mathrm{b}}$ Significant difference between Cardiology and Endocrinology and between Endocrinology and Plastic Surgery.

GLP-1, glucagon-like peptide-1; HbA1c, hemoglobin A1c; OAD, oral antidiabetes drug.

insulin three times a day ( $45 \%$ of the total dose for breakfast bolus, $25 \%$ for lunch bolus, and $30 \%$ for dinner bolus). The total daily dose was adjusted by the treating physician during the ward round. Therefore the basal insulin was administered after the ward round at lunchtime.

The following safety features were implemented into the GlucoTab system: if a patient would not eat, basal insulin was administered, but the prescribed bolus insulin was withheld, and correctional bolus doses were administered for the regulation of particular BG values if required. The GlucoTab system also took into account the amount of bolus insulin that was still active in the patient's body from a previous dose ("insulin on-board"), by reducing bolus insulin by $25 \%$ per hour. ${ }^{15}$ Another safety feature was to reduce the dose of basal insulin if the current basal dose injection was delayed. At any time, the healthcare professionals could overrule the suggested insulin dose and perform additional BG measurements.

At the beginning of the standardized glycemic management, a patient's preexisting antidiabetes therapy with glinides, sulfonylureas, and glitazones was stopped, and patients were assigned to receive standardized glycemic management according to the GlucoTab recommendations. Metformin and/or incretin-based therapies were maintained if there was no contraindication. At discharge, patients returned to their previous antidiabetes treatment, unless the treating physician prescribed continuing the insulin therapy performed during the study or changing to another insulin therapy.

All nurses and physicians were instructed on the study protocol, study-specific procedures, handling the GlucoTab system, and Good Clinical Practice before the start of the study. Healthcare professionals were invited to participate in a workshop about diabetes before study start and to fill out an usability questionnaire at the end of the study.

Capillary BG values were measured by using a point-ofcare testing device (ACCU-CHEK ${ }^{\circledR}$ Inform system; Roche Diagnostics, Rotkreuz, Switzerland), which is integrated into the laboratory quality management system. Capillary BG measurements and insulin dosing were performed and documented by the nurse on duty.

CGM (iPro ${ }^{\mathrm{TM}} 2$; Medtronic, Northridge, CA) data were available for a subset of 35 patients from 42 patients in total on the Endocrinology Ward; one patient lost the sensor, three patients had too few data points for analysis, and for another three patients no sensor transmitter was available. As CGM data were analyzed retrospectively, the treatment was not influenced by these data. 


\section{Statistical analysis}

A sample size calculation was performed in order to test the study hypothesis by using a one-tailed one-sample $t$ test weighted by the total number of BG measurements per subject, with a $5 \%$ level of significance and a power of $95 \%$.

In order to test whether the mean percentage of BG measurements in the target range $70-140 \mathrm{mg} / \mathrm{dL}$ (primary outcome) was greater than the recent best-practice study with the criterion value of $42 \%,{ }^{12}$ we applied an one-tailed onesample $t$ test, weighted by the total number of BG measurements per subject. The level of significance was set to $5 \%$.

The wards were compared by using the nonparametric Kruskal-Wallis rank sum test (metric variables) for analysis of secondary outcomes because patients were unequally distributed among the wards, with some table cells being unacceptably small for an analysis of variance. In case of a significant Kruskal-Wallis test, we performed pairwise comparisons by using the (nonparametric) Mann-Whitney $U$ test. Fisher's exact test was used for nominal scales. No corrections for multiple testing were used, and the level of significance was set to $5 \%$ for all tests.

Finally, a multiple regression model to predict the mean daily BG value over all study days, except study Day 1, was fitted to the data. Study Day 1 was excluded because of incomplete datasets. Variables were sex, age, creatinine, hemoglobin A1c (HbA1c), body mass index, first total daily insulin dose per kilogram of body weight, diabetes duration, preexisting home insulin therapy at admission (yes, no), oral antidiabetes drugs at admission (yes, no), clinical ward, admission type (planned, acute), and the interaction between admission type and clinical ward. Model simplification was performed by using Akaike's information criterion. Statistical analysis was performed using $\mathrm{R}$ version 2.13 .1 software. $^{16}$

Table 2. Efficacy, Safety, and Usability of the Glucotab System on Different General Wards

\begin{tabular}{|c|c|c|c|c|c|}
\hline Variable & $\begin{array}{c}\text { Total } \\
(\mathrm{n}=99)\end{array}$ & $\begin{array}{l}\text { Nephrology } \\
\quad(\mathrm{n}=15)\end{array}$ & $\begin{array}{l}\text { Cardiology } \\
(\mathrm{n}=30)\end{array}$ & $\begin{array}{l}\text { Endocrinology } \\
\qquad(\mathrm{n}=42)\end{array}$ & $\begin{array}{l}\text { Plastic surgery } \\
\quad(\mathrm{n}=12)\end{array}$ \\
\hline Length of study (days) & $7.8 \pm 4.5$ & $8.5 \pm 5.4$ & $6.8 \pm 4.1$ & $8.8 \pm 4.4$ & $5.9 \pm 3.7$ \\
\hline \multicolumn{6}{|l|}{ Implementation (\%) } \\
\hline \multicolumn{6}{|l|}{ Performance of expected } \\
\hline BG measurement & 95.2 & 92.4 & 97.2 & 94.8 & 98.4 \\
\hline Bolus insulin injections & 94.2 & 96.8 & 97.4 & 93.2 & 86.5 \\
\hline Basal insulin injections & 99.4 & 100 & 100 & 98.7 & 100 \\
\hline \multicolumn{6}{|l|}{ Adherence to } \\
\hline Total daily insulin dose & 97.5 & 98.5 & 97.5 & 98.0 & 92.9 \\
\hline Bolus dose suggestion & 96.5 & 94.3 & 97.2 & 96.3 & 95.1 \\
\hline Basal insulin suggestion & 96.7 & 91.1 & 96.3 & 96.8 & 91.0 \\
\hline \multicolumn{6}{|l|}{ Efficacy and safety } \\
\hline \multicolumn{6}{|l|}{ BG $(\mathrm{mg} / \mathrm{dL})$} \\
\hline Mean daily & $154 \pm 35$ & $162 \pm 34$ & $163 \pm 33$ & $150 \pm 35$ & $134 \pm 31$ \\
\hline Mean prebreakfast & $147 \pm 43$ & $151 \pm 38$ & $156 \pm 47$ & $147 \pm 44$ & $119 \pm 28$ \\
\hline Mean prelunch & $170 \pm 54$ & $197 \pm 59$ & $179 \pm 58$ & $163 \pm 50$ & $137 \pm 36$ \\
\hline Mean predinner & $153 \pm 41$ & $141 \pm 51$ & $164 \pm 40$ & $146 \pm 36$ & $164 \pm 42$ \\
\hline Mean bedtime & $153 \pm 39$ & $165 \pm 41$ & $164 \pm 31$ & $146 \pm 39$ & $136 \pm 42$ \\
\hline Pre-enrollment & $188 \pm 73$ & $185 \pm 43$ & $173 \pm 58$ & $204 \pm 88$ & $158 \pm 55$ \\
\hline BG in target $70-140 \mathrm{mg} / \mathrm{dL}(\%)^{\mathrm{a}}$ & $50.2 \pm 22.2$ & $39.3 \pm 13.7$ & $40.7 \pm 18.9$ & $52.3 \pm 20.7$ & $64.9 \pm 24.6$ \\
\hline \multicolumn{6}{|l|}{ BG in different ranges $(\%)$} \\
\hline$<40 \mathrm{mg} / \mathrm{dL}$ & 0.0 & 0.0 & 0.0 & 0.0 & 0.0 \\
\hline 40 to $<60 \mathrm{mg} / \mathrm{dL}$ & 0.5 & 0.2 & 0.0 & 0.8 & 0.4 \\
\hline 60 to $<70 \mathrm{mg} / \mathrm{dL}$ & 1.4 & 0.8 & 0.3 & 2.2 & 1.3 \\
\hline 70 to $<180 \mathrm{mg} / \mathrm{dL}$ & 72.5 & 64.6 & 70.6 & 74.4 & 83.7 \\
\hline 180 to $<300 \mathrm{mg} / \mathrm{dL}$ & 22.9 & 29.4 & 27.2 & 20.0 & 14.2 \\
\hline$\geq 300 \mathrm{mg} / \mathrm{dL}$ & 2.7 & 5.0 & 1.9 & 2.6 & 0.4 \\
\hline \multicolumn{6}{|l|}{ Antihyperglycemic therapy } \\
\hline First calculated TDD (IU) ${ }^{\mathrm{b}}$ & $38.9 \pm 21.7$ & $33.6 \pm 11.3$ & $33.5 \pm 16.6$ & $44.8 \pm 27.5$ & $38.3 \pm 15.1$ \\
\hline First TDD/kg of body weight (IU) & $0.43 \pm 0.19$ & $0.36 \pm 0.10$ & $0.39 \pm 0.11$ & $0.49 \pm 0.24$ & $0.44 \pm 0.14$ \\
\hline \multicolumn{6}{|c|}{ Mean daily injected insulin dose during study (IU) } \\
\hline Injected bolus insulin dose & $28.5 \pm 19.2$ & $27.3 \pm 14.9$ & $25.8 \pm 12.3$ & $32.5 \pm 25.1$ & $21.0 \pm 6.7$ \\
\hline Injected basal insulin dose & $22.9 \pm 18.2$ & $21.0 \pm 7.6$ & $17.8 \pm 8.6$ & $28.7 \pm 25.0$ & $17.1 \pm 7.4$ \\
\hline \multicolumn{6}{|l|}{ Concomitant drugs $(n)$} \\
\hline Patients with OADs & 36 & 2 & 13 & 14 & 7 \\
\hline Patients with GLP-1 analogs & 6 & 0 & 2 & 4 & 0 \\
\hline Patients with steroids & 4 & 1 & 1 & 1 & 1 \\
\hline
\end{tabular}

${ }^{a}$ Primary end point. Significant differences occurred between Endocrinology and Cardiology $(P=0.02)$, Plastic Surgery and Nephrology $(P=0.01)$, Plastic Surgery and Cardiology $(P=0.02)$, and Nephrology and Endocrinology $(P=0.01)$.

${ }^{\mathrm{b}}$ Total daily dose (TDD) might deviate from the injected total insulin dose of Day 1 depending on the time of day when a patient was started on GlucoTab therapy.

BG, blood glucose; GLP-1, glucagon-like peptide-1; IU, international units; OAD, oral antidiabetes drug. 


\section{Results}

Implementation of standardized glycemic management

The standardized workflow support with the GlucoTab system was highly accepted by healthcare professionals on all participating clinical wards as indicated by the performance of the expected BG measurements and the adherence to insulin dose suggestions (Table 2).

In total, physicians adhered to the suggested total daily insulin doses in $97.5 \%$ of cases (Table 2 and Fig. 1), and nurses' adherence rates with suggested bolus insulin doses and basal insulin doses were $96.5 \%$ and $96.7 \%$, respectively. If corrections were performed by healthcare professionals, the changes were relatively small: $0.7 \pm 1.6$ international inits (IU) for bolus insulin and $0.9 \pm 2.8 \mathrm{IU}$ for basal insulin.

\section{Efficacy of standardized glycemic management}

By using the GlucoTab system, the percentage of BG values in the target range increased over time in all participating clinical wards (Fig. 1). Overall, the mean percentage of BG measurements in the target range $70-140 \mathrm{mg} / \mathrm{dL}$ was

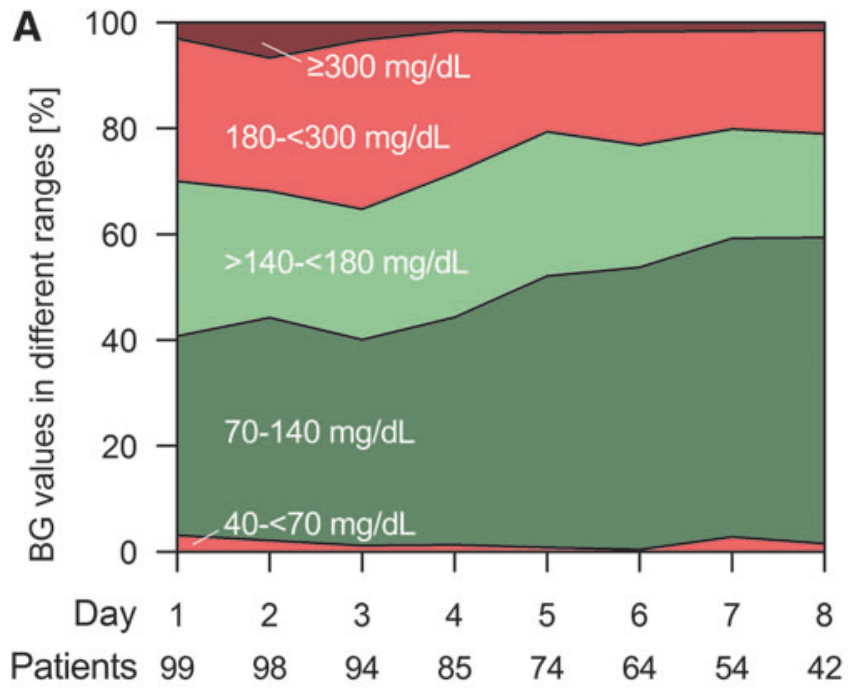

FIG. 1. (A) Mean percentage of premeal and bedtime blood glucose (BG) values in different ranges of standardized glycemic management for patients on different wards. (B) Total, bolus, and basal insulin dose (mean $\pm \mathrm{SE}$ ) of standardized glycemic management for patients on different wards. Insulin dose on Day 1 was lower depending on the time of day when a patient was started on GlucoTab therapy. IU, international units. (C) Comparison of estimated BG based on hemoglobin A1c level at admission with mean BG during hospital stay.
$50.2 \pm 22.2 \%$, which was significantly higher than the criterion value of $42 \%$ deriving from a recent best-practice study $(P=0.001){ }^{12}$ Of the patients, $72.2 \%$ had a reduction of the mean BG during hospital stay compared with the estimated BG based on HbA1c at admission. ${ }^{17}$ In all patients with an estimated average BG of $>200 \mathrm{mg} / \mathrm{dL}$, based on the $\mathrm{HbA} 1 \mathrm{c}$, the mean BG during the study was improved (Fig. 1C). The overall mean of $2,466 \mathrm{BG}$ measurements was $154 \pm 35 \mathrm{mg} /$ dL. Details of glycemic management across the clinical wards are shown in Table 2.

The percentage of BG in the target range $70-140 \mathrm{mg} / \mathrm{dL}$ was highest on the Plastic Surgery Ward $(64.9 \pm 24.6 \%)$. The lowest value was found on the Nephrology Ward $(39.3 \pm 13.7 \%)$. Analysis of the CGM data of patients on the Endocrinology Ward indicated that more than half of the study time $(54.0 \%)$ subcutaneous BG values were in the target range of $70-140 \mathrm{mg} / \mathrm{dL}$ (Fig. 2) and confirmed that reference $\mathrm{BG}$ values were representative $(52.3 \%$ in the target range; Table 2).

Although these observations suggest variations within glycemic management among the clinical wards, a regression analysis to predict the mean daily BG value showed that the
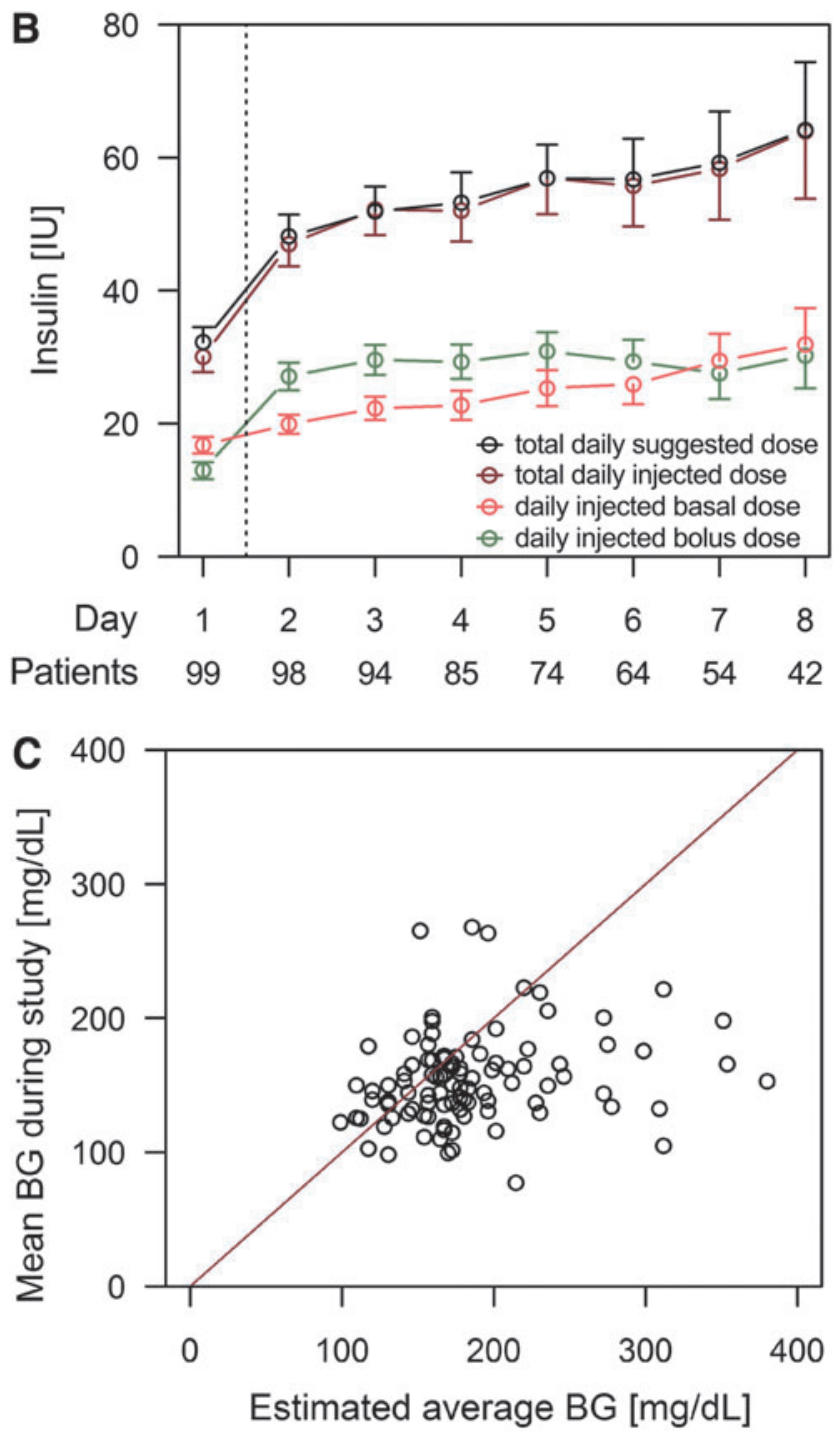


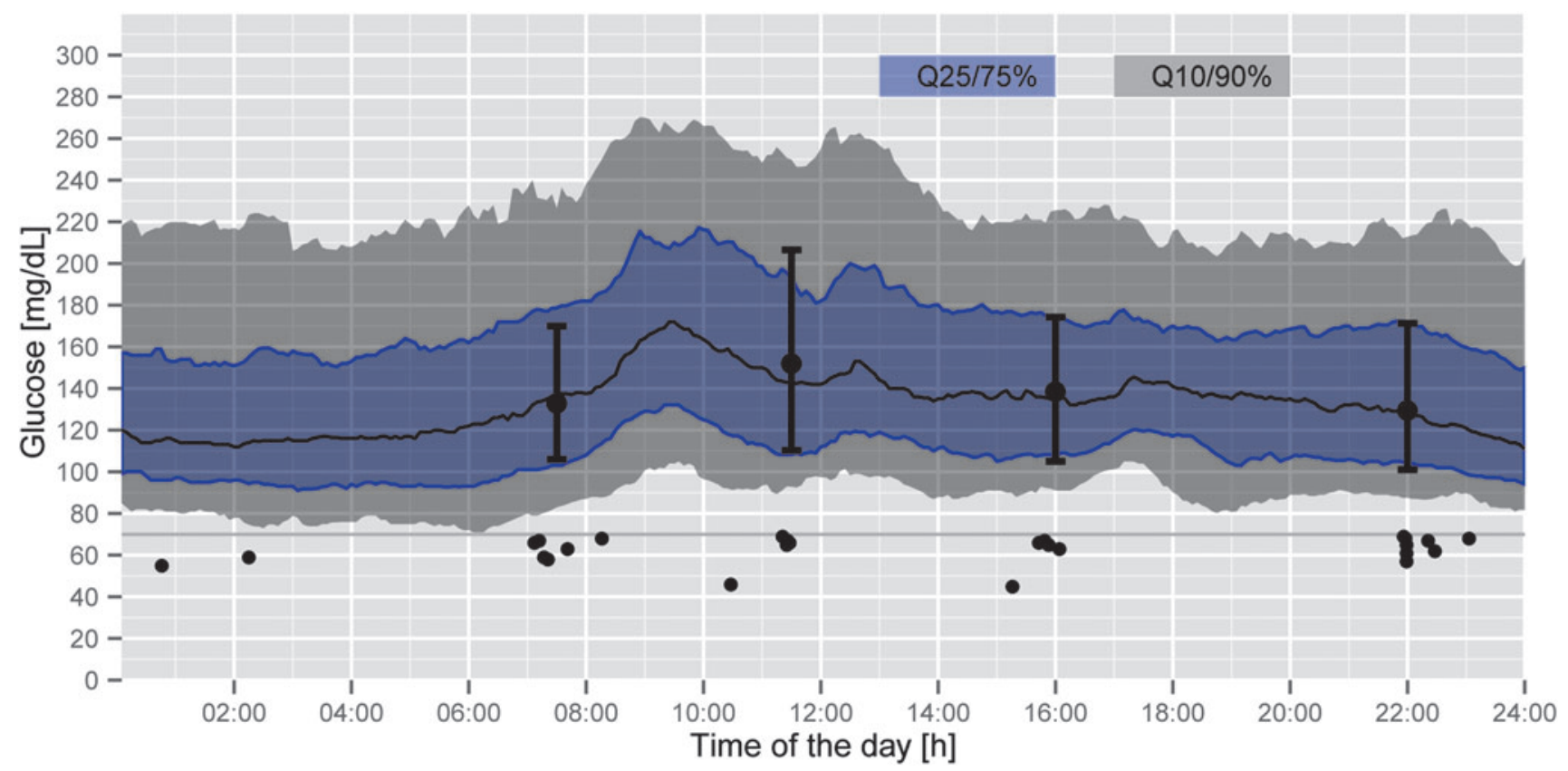

FIG. 2. Daily continuous glucose monitoring profiles and reference blood glucose values (black circles $=$ reference blood glucose values) in 35 patients on the Endocrinology Ward. Q, quartile.

BG value was not affected by a specific clinical ward. Patients with preexisting home insulin therapy at admission had, on average, higher $(+26 \mathrm{mg} / \mathrm{dL})$ mean daily $\mathrm{BG}$ values during the standardized glycemic management than patients without preexisting home insulin therapy. Particularly on the Cardiology Ward the type of admission had a strong impact on the mean daily BG value. Acutely admitted patients on the Cardiology Ward had on average higher $(+30 \mathrm{mg} / \mathrm{dL})$ mean daily $\mathrm{BG}$ values than patients with acute admissions at the other wards $(+4 \mathrm{mg} / \mathrm{dL})$. Furthermore, the regression analysis showed that a higher first insulin dose per kilogram of body weight by $0.1 \mathrm{IU}$ or a lower $\mathrm{HbA} 1 \mathrm{c}$ value at admission by $10 \mathrm{mmol} / \mathrm{mol}$ was associated with a lower mean BG by $5 \mathrm{mg}$ / $\mathrm{dL}$ and $4 \mathrm{mg} / \mathrm{dL}$, respectively.

\section{Safety of standardized glycemic management}

The number of hypoglycemic events on the different wards using the GlucoTab system was comparable (Table 2). No severe hypoglycemic event below $40 \mathrm{mg} / \mathrm{dL}$ was observed. Of all measurements in the range 40 to $<60 \mathrm{mg} / \mathrm{dL}, 0.5 \%$ occurred in nine different patients. Of the measurements in the range of 60 to $<70 \mathrm{mg} / \mathrm{dL}, 1.4 \%$ occurred in 24 different patients. In patients on the Endocrinology Ward, the analysis of the CGM data confirmed a low risk for developing hypoglycemia: $0 \%$ and $1.2 \%$ for measurements in the ranges $<40 \mathrm{mg} / \mathrm{dL}$ and 40 to $<60 \mathrm{mg} / \mathrm{dL}$, respectively (Fig. 2).

Twenty-eight mild and moderate adverse events and one serious adverse event (stent thrombosis) occurred. None of these events was recognized as related to the GlucoTab system.

\section{Usability of standardized glycemic management}

At the end of the study, 65 healthcare professionals completed a questionnaire (54 women and 11 men; mean age, $36 \pm 11$ years; 51 nurses, 14 physicians). Forty-two health- care professionals had previous experience with the use of mobile devices. Fifty-nine healthcare professionals (91\%) felt confident in performing glycemic management with the GlucoTab system. Fifty-eight healthcare professionals (89\%) believed that the system was practical to use in daily clinical routine. Fifty-two participants $(80 \%)$ stated that using GlucoTab could prevent medical errors associated with drug prescriptions. Fifty-six healthcare professionals (86\%) answered that when using the system, physicians had to be consulted less often about glycemic management. Fifty-five healthcare professionals $(85 \%)$ stated that glycemic control was more efficient when using the GlucoTab system. Different perceptions of workload were assessed. Thirteen healthcare professionals indicated a workload increase, 33 indicated a workload decrease, and 12 indicated no change in the workload, when using the GlucoTab system. Seven healthcare professionals did not answer this question.

\section{Discussion}

Our data indicate that standardized glycemic management guided by the GlucoTab system for workflow and decision support can be implemented efficiently and safely and is useraccepted in different wards in a tertiary-care hospital. Of the BG measurements, $50.2 \pm 22.2 \%$ were in the target range (70$140 \mathrm{mg} / \mathrm{dL}$ ) by using the GlucoTab. Moreover, the system was implemented without any occurrence of severe hypoglycemia and with a high acceptance rate among healthcare professionals.

The high number of BG measurements and insulin injections performed according to suggested standardized care showed that the GlucoTab system was highly accepted and continuously used by healthcare professionals and that it was able to successfully guide the glycemic management process. This was also confirmed by the user questionnaire and by the tight adherence of healthcare professionals to the suggested 
insulin doses. Adherence of healthcare professionals was considerably higher than in previous studies. ${ }^{18,19}$ Schnipper et al. ${ }^{19}$ performed a study on computerized order sets and reported that $67 \%$ of the patients received an adequate initial dose of nutritional insulin and that in only $37 \%$ of the patients' insulin orders were changed.

In our study, differences regarding glycemic control among the wards were observed, although adherence rates to insulin dosing and BG measurement suggestions of the GlucoTab system were comparable on the participating clinical wards. However, a regression analysis revealed that ward assignment was not an appropriate variable to predict the mean BG value. In this analysis, an acute admission influenced the glycemic control on all wards, and the strongest influence of an acute admission on BG was found on the Cardiology Ward $(+30 \mathrm{mg} / \mathrm{dL})$. We assume that these findings may be related to myocardial infarction, which is the predominant diagnosis of acute admission on the Cardiology Ward and which is associated with local and systemic inflammation ${ }^{20}$ leading to impaired glycemic control and possible linkage to poor cardiac outcome. ${ }^{21}$

According to our regression analysis, further modification of the basal bolus algorithm may be required for a more personalized care in the acute phase of cardiac events.

Higher mean BG measurements were observed not only on the Cardiology Ward, but also on the Nephrology Ward. It is surprising that the creatinine value did not influence the mean daily $B G$ value according to the regression analysis. Thus, we assume that the lower first total daily insulin dose may be responsible for the less stringent glycemic control in patients on the Nephrology Ward. This lower first total insulin dose was a strong predictor for impaired glycemic control in the model. According to the algorithm design, the initial dose was reduced from 0.5 to $0.3 \mathrm{IU} / \mathrm{kg}$ of body weight if a creatinine value was $>2 \mathrm{mg} / \mathrm{dL}$. However, a recent randomized controlled trial in patients with a glomerular filtration rate of $<45 \mathrm{~mL} / \mathrm{min}$ showed that an insulin starting dose of $0.25 \mathrm{IU} /$ $\mathrm{kg}$ of body weight did not worsen glycemic control when compared with the control group that used an insulin starting dose of $0.5 \mathrm{IU} / \mathrm{kg}$ of body weight. The authors speculated that in these patients the insulin resistance might be a key element of impaired glycemic control. ${ }^{22}$

According to the regression analysis, preexisting home insulin therapy and the HbAlc values in addition to the type of hospital admission and the first total daily insulin dose are essential factors that influence the mean BG values during hospitalization. Thus, these factors have the potential to be used for a more personalized algorithm.

In our study the risk of hypoglycemia was low. None of the BG values was below $40 \mathrm{mg} / \mathrm{dL}$. Hypoglycemic events were evenly distributed among patients. The percentages of BG measurements in the different hypoglycemic ranges and in the target range were similar to those found in comparable studies. $11,12,23,24$

Several limitations of our study have to be addressed. The present study was a noncontrolled clinical study. However, a retrospective assessment of glycemic control on two wards participating in this study achieved 57\% (Endocrinology) and $51 \%$ (Cardiology) in the range of $70-180 \mathrm{mg} / \mathrm{dL}$ in routine care. In addition, the results of a previously published prospective controlled study on these two wards showed that patients in a paper-based basal bolus algorithm group had a significantly higher percentage of BG measurements in the range of $70-180 \mathrm{mg} / \mathrm{dL}$ than patients in routine care group (73\% vs. 53\%). These data indicate that glycemic control was improved by the use of the GlucoTab system compared with routine care. ${ }^{7,13}$

Because of the competitive recruiting process, the number of included patients per ward differed considerably, and results from the different wards and the regression analysis can only be interpreted with caution. The implemented target range of less than $140 \mathrm{mg} / \mathrm{dL}$ for premeal BG measurements may have to be reconsidered for certain populations in hospital care. Modified algorithms (e.g., for geriatric patients with individualized target ranges ${ }^{25}$ ) need to be developed and evaluated.

In conclusion, our data demonstrate that the GlucoTab system allowed an efficacious, safe, and user-accepted implementation of standardized glycemic management in different general wards of a tertiary-care hospital. Consequently, the system can support healthcare professionals in improving glycemic management relying on evidence-based guidelines for non-critically ill hospitalized patients.

\section{Acknowledgments}

The authors acknowledge the critical review of the manuscript and the editorial assistance of B. Boulgaropoulos, Medical University of Graz, the data management of B. Tschapeller, C. Krainer (both from Joanneum Research $\mathrm{GmbH}$ ), and G. Schwagerle, Medical University of Graz, the data monitoring of A. Berghofer, Medical University of Graz, the technical implementation of support and software testing of F. Chiarugi and D. Manousos, FORTH-ICS, and the mobile device security and user management of M. Enzmann and F. Franke, Fraunhofer SIT. The authors thank the nurses and physicians of the Divisions of Endocrinology and Metabolism, Cardiology, Nephrology, and Plastic Surgery, Medical University of Graz, for their contribution in the performance of the study. This study was performed within the REACTION project, which was funded by the European Commission under the $7^{\text {th }}$ Framework Program in the area of Personal Health Systems under Grant Agreement Number 248590.

\section{Author Disclosure Statement}

T.R.P. is a member in the advisory board of NovoNordisk A/S, Eli Lilly, and Roche Diagnostics and has received speaker honoraria from NovoNordisk A/S, Eli Lilly, and AstraZeneca. J.K.M. is a member in the advisory board of Sanofi-Aventis Austria and has received speaker honoraria from NovoNordisk A/S and Roche Diagnostics. J.P. has received speaker honoraria from NovoNordisk A/S. K.M.N., B.H., F.A., K.D., T.A., L.S., S.S., P.B., F.M., C.S., A.R.R., D.B.L., and L.-P.K. declare no competing financial interests exist.

K.M.N., J.K.M., B.H., S.S., P.B., L.S., and J.P. designed and performed the study, interpreted data, and contributed to discussions. K.M.N. drafted the manuscript. T.A. and K.D. designed the study and performed statistical analysis. F.A., C.S., and D.B.L. performed the study. A.R.R., F.M.F., and L.-P.K. supervised the project. T.R.P. interpreted data, contributed to discussions, supervised the project, and is the guarantor of this work. All authors critically revised the article and approved the final version of the manuscript. 


\section{References}

1. Schaupp L, Donsa K, Neubauer KM, et al.: Taking a closer look-continuous glucose monitoring in non-critically ill hospitalized patients with type 2 diabetes mellitus under basal-bolus insulin therapy. Diabetes Technol Ther 2015; 17:000-000.

2. Umpierrez GE: Hyperglycemia: an independent marker of in-hospital mortality in patients with undiagnosed diabetes. J Clin Endocrinol Metab 2002;87:978-982.

3. Rayman G, Service NH: National Diabetes Inpatient Audit 2013. 2014. www.hscic.gov.uk/catalogue/PUB13662/ natidiab-inp-audi-13-nat-rep.pdf (accessed November 25, 2014).

4. American Diabetes Association: Economic costs of diabetes in the U.S. in 2007. Diabetes Care 2008;31:596-615.

5. Murad MH, Coburn JA, Coto-Yglesias F, et al.: Glycemic control in non-critically ill hospitalized patients: a systematic review and meta-analysis. J Clin Endocrinol Metab 2012;97:49-58.

6. Draznin B, Gilden J, Golden SH, et al.: Pathways to quality inpatient management of hyperglycemia and diabetes: a call to action. Diabetes Care 2013;36:1807-1814.

7. Neubauer KM, Schaupp L, Plank J, et al.: Failure to control hyperglycemia in noncritically ill diabetes patients despite standard glycemic management in a hospital setting. J Diabetes Sci Technol 2013;7:402-409.

8. American Diabetes Association: Standards of medical care in diabetes - 2015. Diabetes Care 2015;38(Suppl 1):S9-S99.

9. Umpierrez GE, Hellman R, Korytkowski MT, et al.: Management of hyperglycemia in hospitalized patients in non-critical care setting: an Endocrine Society clinical practice guideline. J Clin Endocrinol Metab 2012;97:16-38.

10. Umpierrez GE, Smiley D, Zisman A, et al.: Randomized study of basal-bolus insulin therapy in the inpatient management of patients with type 2 diabetes (RABBIT 2 trial). Diabetes Care 2007;30:2181-2186.

11. Umpierrez GE, Smiley D, Jacobs S, et al.: Randomized study of basal-bolus insulin therapy in the inpatient management of patients with type 2 diabetes undergoing general surgery (RABBIT 2 surgery). Diabetes Care 2011; 34:256-261.

12. Umpierrez GE, Smiley D, Hermayer K, et al.: Randomized study comparing a basal-bolus with a basal plus correction insulin regimen for the hospital management of medical and surgical patients with type 2 diabetes: basal plus trial. Diabetes Care 2013;36:2169-2174.

13. Mader JK, Neubauer KM, Schaupp L, et al.: Efficacy, usability and sequence of operations of a workflow-integrated algorithm for basal-bolus insulin therapy in hospitalized type 2 diabetes patients. Diabetes Obes Metab 2013;16: 137-146.

14. Mader J, Neubauer K, Aberer F, et al.: Basal bolus insulin therapy in hospitalised patients with diabetes mellitus type
2 using two algorithms embedded in a tablet PC [abstract]. Endocr Abstr 2014;35:P488.

15. Walsh J, Roberts R, Varma C: Using Insulin: Everything You Need for Success with Insulin. San Diego, CA: Torrey Pines Press, 2003.

16. R Development Core Team: R: A language and Environment for Statistical Computing. 2008. www.r-project.org (accessed November 27, 2014).

17. Nathan DM, Kuenen J, Borg R, et al.: Translating the A1C assay into estimated average glucose values. Diabetes Care 2008;31:1473-1478.

18. Wexler DJ, Shrader P, Burns SM, et al.: Effectiveness of a computerized insulin order template in general medical inpatients with type 2 diabetes: a cluster randomized trial. Diabetes Care 2010;33:2181-2183.

19. Schnipper JL, Liang CL, Ndumele CD, et al.: Effects of a computerized order set on the inpatient management of hyperglycemia: a cluster-randomized controlled trial. Endocr Pract 2010;16:209-218.

20. Mulvihill NT, Foley JB: Inflammation in acute coronary syndromes. Heart 2002;87:201-204.

21. Marfella R, Siniscalchi M, Esposito K, et al.: Effects of stress hyperglycemia on acute myocardial infarction: role of inflammatory immune process in functional cardiac outcome. Diabetes Care 2003;26:3129-3135.

22. Baldwin D, Zander J, Munoz C, et al.: A randomized trial of two weight-based doses of insulin glargine and glulisine in hospitalized subjects with type 2 diabetes and renal insufficiency. Diabetes Care 2012;35:1970-1974.

23. Umpierrez GE, Gianchandani R, Smiley D, et al.: Safety and efficacy of sitagliptin therapy for the inpatient management of general medicine and surgery patients with type 2 diabetes: a pilot, randomized, controlled study. Diabetes Care 2013;36:3430-3435.

24. Nirantharakumar K, Chen YF, Marshall T, et al.: Clinical decision support systems in the care of inpatients with diabetes in non-critical care setting: systematic review. Diabet Med 2012;29:698-708.

25. Moreno G, Mangione CM, Kimbro L, et al.: Guidelines abstracted from the American Geriatrics Society Guidelines for improving the care of older adults with diabetes mellitus: 2013 update. J Am Geriatr Soc 2013;61:2020-2026.

Address correspondence to: Julia K. Mader, MD

Division of Endocrinology and Metabolism Department of Internal Medicine Medical University of Graz. Auenbruggerplatz 15 A-8036 Graz, Austria

E-mail: julia.mader@medunigraz.at 